\title{
Process-microstructure-properties relationship in Al-CNTs-Al2O3 nanocomposites manufactured by hybrid powder metallurgy and microwave sintering process
}

\begin{abstract}
Al-2CNTs-xA12O3 nanocomposites were manufactured by a hybrid powder metallurgy and microwave sintering process. The correlation between process-induced microstructural features and the material properties including physical and mechanical properties as well as ultrasonic parameters was measured. It was found that physical properties including densification and physical dimensional changes were closely associated with the morphology and particle size of nanocomposite powders. The maximum density was obtained by extensive particle refinement at milling time longer than $8 \mathrm{~h}$ and Al2O3 content of $10 \mathrm{wt} . \%$. Mechanical properties were controlled by $\mathrm{A} 12 \mathrm{O} 3$ content, dispersion of nano reinforcements and grain size. The optimum hardness and strength properties were achieved through incorporation of $10 \mathrm{wt} . \%$ Al2O3 and homogenous dispersion of CNTs and A12O3 nanoparticles (NPs) at $12 \mathrm{~h}$ of milling which resulted in the formation of high density of dislocations and extensive grain size refinement. Also both longitudinal and shear velocities and attenuation increase linearly by increasing A12O3 content and milling time. The variation of ultrasonic velocity and attenuation was attributed to the degree of dispersion of CNTs and Al2O3 and also less inter-particle spacing in the matrix. The larger A12O3 content and more homogenous dispersion of CNTs and Al2O3 NPs at longer milling time exerted higher velocity and attenuation of ultrasonic wave.
\end{abstract}

Keyword: Hybrid composite; Aluminum composites; Powder metallurgy; Microwave sintering; Microstructure; Mechanical properties; Ultrasonic velocity; Ultrasonic attenuation 